\title{
Therapies in flux: highlights of the 2019 San Antonio Breast Cancer Symposium (SABCS)
}

\author{
Simon Peter Gampenrieder (D)
}

The San Antonio Breast Cancer Symposium (SABCS) in Texas, USA, is an annual highlight for breast cancer researchers from all over the world. Nearly every year, practice-changing clinical trials or meta-analyses are presented at this focused international meeting. In addition to these large studies, the meeting distinguishes itself by excellent scientific and educational sessions as well as a large poster session displaying the many-sided facets of breast cancer over five consecutive days.

In this issue of memo-magazine of european medical oncology, the most important studies for clinical practice presented at the $42 \mathrm{nd}$ annual SABCS in December 2019 are summarized in short reviews by three distinct experts in the field. As a reader, you will gain an overview of the main themes of the meeting, irrespective of whether you could or could not attend the symposium in Texas personally.

Similar to the years before, studies in HER2(Human Epidermal Growth Factor Receptor 2)-positive breast cancer constituted the highlights of the 2019 congress. The randomized phase II trial HER2CLIMB [1] investigated tucatinib, a new and highly selective inhibitor of the HER2 tyrosine kinase, in combination with trastuzumab and capecitabine in metastatic HER2positive breast cancer. Despite extensive pretreatment and a high rate of patients with brain metastases, the

\footnotetext{
S. P. Gampenrieder $(\bowtie)$

Department of Internal Medicine III with Haematology,

Medical Oncology, Haemostaseology, Infectiology and Rheumatology, Oncologic Center, Salzburg Cancer Research Institute-Laboratory for Immunological and Molecular Cancer Research (SCRI-LIMCR), Paracelsus Medical University Salzburg, Müllner Hauptstraße 48, 5020 Salzburg, Austria

Cancer Cluster Salzburg, Salzburg, Austria s.gampenrieder@salk.at
}

new compound significantly improved the hazard ratio for disease progression or death. Given the fact that brain metastases still represent one of the major problems in this disease, the high intracranial activity of this compound adds significant breadth to our treatment arsenal for HER2-positive breast cancer.

In the same congress session, the multicenter, single-group, phase 2 study DESTINY-Breast01 was presented [2]. Patients with HER2-positive metastatic breast cancer pretreated with multiple therapy lines (median six, range two to 27) including T-DM1, received the drug-antibody conjugate trastuzumab deruxtecan and experienced clinical response and disease control in $61 \%$ and $76 \%$ of cases, respectively. Unfortunately, $14 \%$ of patients developed interstitial lung disease (ILD), which was lethal in four patients (2.2\%). Particularly this side effect requires careful monitoring and further investigation in the running phase 3 trials.

In addition to these breakthrough studies, an update on key subgroups of the Keynote-522 trial was presented [3]. This pioneering phase III trial for immunotherapy in early triple-negative breast cancer investigated the anti-PD-1 antibody pembrolizumab in combination with standard chemotherapy in the neoadjuvant setting. After surgery, patients continued to receive pembrolizumab or placebo for a total of 1 year. Of note, this study was the first neoadjuvant trial powered to investigate the combined primary endpoint of pathologic complete response (pCR) and event-free survival (EFS). Furthermore, a validation of the Clinical Treatment Score Post 5 Years (CTS5) was presented [4], and the role of capecitabine as part of (neo)adjuvant treatment was clarified [5, 6]. Prevention trials with anastrozole (IBIS-II trial) [7] or low-dose tamoxifen (TAM01 trial) [8] showed positive results, while four trials failed to demonstrate fea- 
sibility of post-neoadjuvant image-guided biopsy to detect pCR [9-12].

If this short teaser awakens your interest, stay tuned and keep reading the following reviews, which summarize these and several more trials presented at last year's symposium. Maybe, next time, on your way to San Antonio, you will ask yourself whether the convenience and efficiency of good reviews do not outweigh the burden of a long journey to Texas.

Conflict of interest S.P. Gampenrieder: Employment: none; Leadership: none; Stock or other Ownership: none; Honoraria: Novartis, Roche, BMS, AstraZeneca, MSD; Consulting or Advisory Role: Roche, Novartis,' Pfizer, Lilly, AstraZeneca, MSD; Speakers Bureau: none; Research Funding: Roche; Patents, Royalties or Other Intellectual Property: none; Expert Testimony: none; Travel, Accommodation, Expenses: Roche, Amgen, Shire, Novartis, Pfizer, Bayer, Celgene, Daiichi Sanky; Other Relationships: none.

\section{References}

1. Murthy R, et al. Abstract GS1-01: Tucatinib vs placebo, both combined with capecitabine and trastuzumab, for patients with pretreated HER2-positive metastatic breast cancer with and without brain metastases (HER2CLIMB). Cancer Res. 2020;80(4 Supplement):GS1-01.

2. Krop IE, et al. Abstract GS1-03: [Fam-] trastuzumab deruxtecan (T-DXd; DS-8201a) in subjects with HER2-positive metastatic breast cancer previously treated with T-DM1: A phase 2, multicenter, open-label study (DESTINYBreast01). Cancer Res. 2020;80 (4 Supplement):GS1-03.

3. Schmid P, et al. Abstract GS3-03: KEYNOTE-522 study of neoadjuvant pembrolizumab + chemotherapy vs placebo + chemotherapy, followed by adjuvant pembrolizumab vs placebo for early triple-negative breast cancer: pathologic complete response in key subgroups and by treatment exposure, residual cancer burden, and breast-conserving surgery. Cancer Res. 2020;80(4 Supplement):GS3-03.

4. Sestak I, et al. Abstract GS4-03: Validation of the clinical treatment score post 5 years (CTS5) in women with hormone receptor positive, HER2-negative, node-negative disease from the TAILORx study. Cancer Res. 2020;80(4 Supplement):GS4-03.

5. Li J, et al. Abstract GS1-08: Adjuvant capecitabine in combination with docetaxel and cyclophosphamide plus epirubicin for triple-negative breast cancer (cbcsg010): An open-label, randomised, multicentre, phase 3 trial. Cancer Res. 2020;80(4Supplement):GS1-08.

6. van Mackelenbergh M, et al. Abstract GS1-07: Effects of capecitabine as part of neo-/adjuvant chemotherapy. A meta-analysis of individual patient data from 12 randomized trials including 15,457 patients. Cancer Res. 2020;80(4 Supplement):GS1-07.

7. Cuzick J, et al. Abstract GS4-04: Ten year results of the international breast cancer intervention study II. Cancer Res. 2020;80(4Supplement):GS4-04.

8. De Censi A. Abstract ES3-3: Low dose tamoxifen and other approaches to improve chemoprevention uptake. Cancer Res. 2020;80(4 Supplement):ES3-3.

9. Heil J, et al. Abstract GS5-03: Diagnosing residual disease and pathologic complete response after neoadjuvant chemotherapy in breast cancer patients by image-guided vacuum-assisted breast biopsy: Results of a prospective multicenter trial. Cancer Res. 2020;80(4 Supplement):GS5-03.

10. Tasoulis MK, et al. Abstract GS5-04: Accuracy of postneoadjuvant chemotherapy image-guided breast biopsy to predict the presence of residual cancer: A multi-institutional pooled analysis. Cancer Res. 2020;80(4 Supplement):GS5-04.

11. Peeters M-JTFDV, et al. Abstract GS5-06: Towards omitting breast surgery in patients with a pathologic complete response after neoadjuvant systemic treatment: interim analysis of the MICRA trial (Minimally Invasive Complete Response Assessment). Cancer Res. 2020;80(4 Supplement):GS5-06.

12. Basik M, et al. Abstract GS5-05: Primary analysis of NRGBR005, a phase II trial assessing accuracy of tumor bed biopsies in predicting pathologic complete response (pCR) in patients with clinical/radiological complete response after neoadjuvant chemotherapy (NCT) to explore the feasibility of breast-conserving treatment without surgery. Cancer Res. 2020;80(4 Supplement):GS5-05.

Publisher's Note Springer Nature remains neutral with regard to jurisdictional claims in published maps and institutional affiliations.

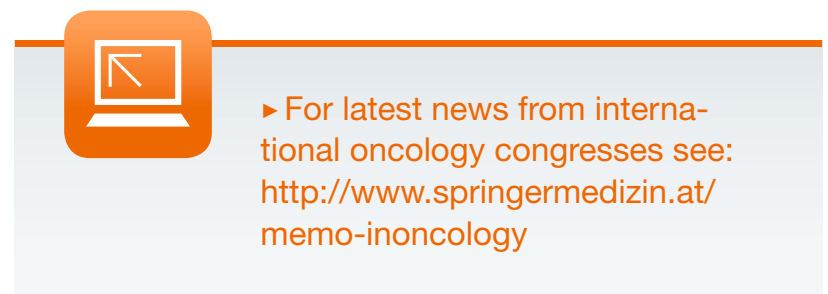

